\title{
Clustering transitions in vibro-fluidized magnetized granular materials
}

\author{
Daniel L. Blair and A. Kudrolli \\ Department of Physics, Clark University, Worcester, MA 01610, USA
}

(October 30, 2018)

\begin{abstract}
We study the effects of long range interactions on the phases observed in cohesive granular materials. At high vibration amplitudes, a gas of magnetized particles is observed with velocity distributions similar to non-magnetized particles. Below a transition temperature compact clusters are observed to form and coexist with single particles. The cluster growth rate is consistent with a classical nucleation process. However, the temperature of the particles in the clusters is significantly lower than the surrounding gas, indicating a breakdown of equipartition. If the system is quenched to low temperatures, a meta-stable network of connected chains self-assemble due to the anisotropic nature of magnetic interactions between particles.
\end{abstract}

Vibro-fluidized inelastic spherical particles are an important model system which capture the essence of dissipative interactions on the statistical properties of dry non-cohesive granular materials [1]. This system has emerged as an important test bed to investigate the applicability of dissipative kinetic theory [2,3]. Experiments measuring the position and velocity of individual particles show the formation of clusters, non-Gaussian velocity distributions (to varying extent), and the violation of equipartition [4:5]. In a number of applications, additional cohesive interactions often exist due to the presence of moisture, electrostatic screening, and magnetization [6].

In this Letter, we introduce a novel system consisting of magnetized particles inside a vibrated container. This enables us to study the effect of long-range attractive interactions on the formation of clusters and the velocity distributions of cohesive granular materials. Non-magnetic particles are also present in our system to define a system temperature that depends on the vibration amplitude of the container. A gas-like phase of magnetized particles is observed at high system temperature. If the system is slowly cooled below a transition temperature, compact clusters precipitate and grow in time. If the temperature is rapidly quenched, an extended mesh of particles is observed to self-assemble due to the anisotropy of the interaction. The structure and growth of the clusters is consistent with both a classical nucleation process, and recent theories of the dipolar hard sphere model [7 [10]. However, the velocity distributions and the associated granular temperature of the free and clustered particles show the influence of dissipation and cohesion.

The idealized interaction between two dipolar hard spheres separated by distance $r$ is defined as

$$
U=U_{H S}+\frac{1}{r^{3}}\left(\overrightarrow{\mu_{i}} \cdot \overrightarrow{\mu_{j}}\right)-\frac{3}{r^{5}}\left(\overrightarrow{\mu_{i}} \cdot \vec{r}_{i j}\right)\left(\overrightarrow{\mu_{j}} \cdot \vec{r}_{i j}\right),
$$

where $U_{H S}$ corresponds to the hard core repulsion interaction, $\vec{\mu}$ is the dipole moment, and $\vec{r}$ is the interparticle vector connecting the centers of dipoles $i, j$. This type of interaction has been put forth as an extremum model for ferro-fluids [1],12. If the potential is averaged over all possible dipole arrangements, the familiar $r^{-6}$ van der Waals interaction is recovered. This implies that such systems should have a well defined liquid gas transition. However, extensive simulation and theoretical work on the dipolar hard sphere model have not conclusively determined the existence of critical phase transition. Recent experiments on ferro-colloid suspensions suggest the existence of a liquid gas transition, but open questions still remain 13. Other experiments using nickel coated glass micro-spheres in a liquid solution have found the existence of ring and mesh-like states [14].

The particles used in our experiments are chrome steel spheres with a diameter of $\sigma=0.3 \mathrm{~cm}$ (with a high degree of sphericity $\delta \sigma / \sigma \sim 10^{-4}$ ) and mass $m=0.12 \mathrm{~g}$. Each sphere has been placed in a ramped field of $1 \times 10^{4} \mathrm{G}$ to embed a permanent moment of $\mu \sim 10^{-2}$ emu per particle. The apparatus consists of a $30.0 \mathrm{~cm}$ diameter flat, anodized aluminum plate, with $1.0 \mathrm{~cm}$ sidewalls and a clear acrylic lid, that is weakly coupled through a rigid linear bearing to an electromechanical shaker. The system is leveled to within $0.001 \mathrm{~cm}$ to ensure that the plate is uniformly accelerated. The measured acceleration of the plate $\Gamma=\mathcal{A} \omega^{2} / g$, where $\mathcal{A}, \omega$ are the amplitude and angular frequency and $g$ is the acceleration due to gravity, is varied between $\Gamma=0-3.0 \mathrm{~g}$, at $\omega=377 \mathrm{rad} \mathrm{s}^{-1}$. Image data is acquired through a high speed Kodak SR1000 digital camera with a spatial (temporal) resolution of $512 \times 480$ pixels (250 f.p.s.). The covering fraction of magnetic particles, $\phi$, defined as the ratio of the area of the particles to that of a close packed mono-layer, is varied from $\phi=0.01 \rightarrow 0.15$. We also place glass particles of equivalent mass, with a fixed volume fraction of $\phi_{p}=0.15$ to introduce additional stochasticity into the system. The glass particles act as thermal carriers to ensure that as the magnetic particles condense, the temperature of the system does not go to zero.

The granular temperature is defined as $T_{g}=\frac{1}{2} m\left\langle\mathbf{v}^{2}\right\rangle$. By measuring velocity components along the two horizontal axes $\mathbf{v}_{x, y}$ we build a distribution of velocities whose width, given by the second moment, determines the granular temperature. The form of the $\mathbf{v}_{x, y^{-}}$ distributions deviate from a Gaussian consistent with 


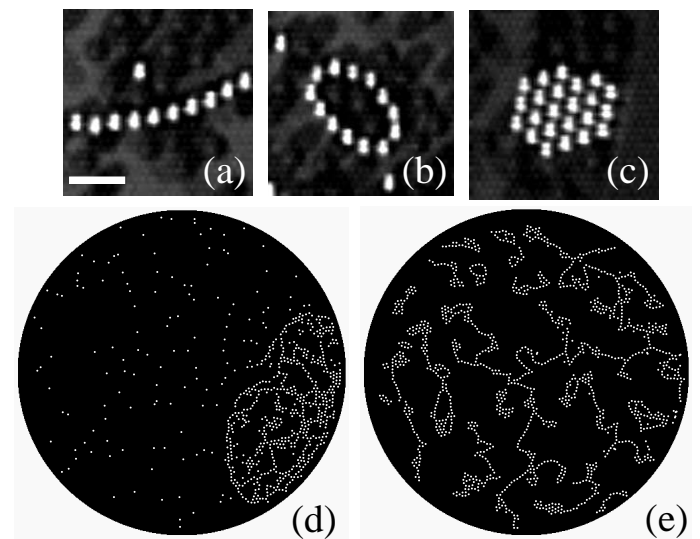

FIG. 1. Examples of chains (a), rings (b), and crystallites (c) observed. Rings appear be the most stable configuration. The scale bar denotes $1 \mathrm{~cm}$. (d) Snapshot of the system at $\phi=0.09$, where $T$ is lowered to $T_{s}$ from the gas state after $1092 \mathrm{~s}$. (c) The system at $\phi=0.15$ after a rapid quench from the gas state into the network state. Images $(\mathrm{c}, \mathrm{d})$ are the size of the system.

previous observations [4]. The system temperature $T$, is determined by the velocity distribution of the gas of both magnetic and non-magnetic particles. We will return to a more detailed analysis of the velocity distributions for the gas and clustered phases after discussing the nature of the clusters.

Our experiments begin by maintaining a gas of magnetic and glass particles at a particular $\phi$. By lowering $T$, a fraction of the magnetic particles begin to self-assemble into the simple structures shown in Fig. 11 $(\mathrm{a}-\mathrm{c})$. We define the temperature at which this occurs as the transition temperature, $T_{s}$. These structures are transient, and over time will evolve into large clusters that coexist in steady state with single particles. An example where a single ring has grown in time to form an extended cluster, is shown in Fig. 11(d) [15]. However, clusters of a very different conformation are produced if $T$ is quenched far below $T_{s}$. Figure 1(e) is an example of the system after a rapid quench. This extended mesh will eventually rearrange to take on more a compact form, therefore we consider this phase to be a meta-stable version of the clustered phase.

To characterize the gas and clustered phases observed, and the nature of the transitions, we plot the phase diagram [Fig. 2] [16]. The connected line shows the transition temperature $T_{s}$ as a function of $\phi$. Above $T_{s}$, short lived dimers and trimers are observed along with single particles, but at and below this temperature, small seeds of magnetic particles [Fig. 1 $(\mathrm{a}-\mathrm{c})$ ], precipitate from the gas. Initially seeds take on the form of short chains that quickly become either rings or more compact states (it is noteworthy to mention that rings $\geq 4$ particles are energetically more favorable than a chain of equal particle number 12,14]). Figure 1 (d) shows an image of the system with $\phi=0.09$ after $1092 \mathrm{~s}$ at $T_{s}$. If $T$ is increased

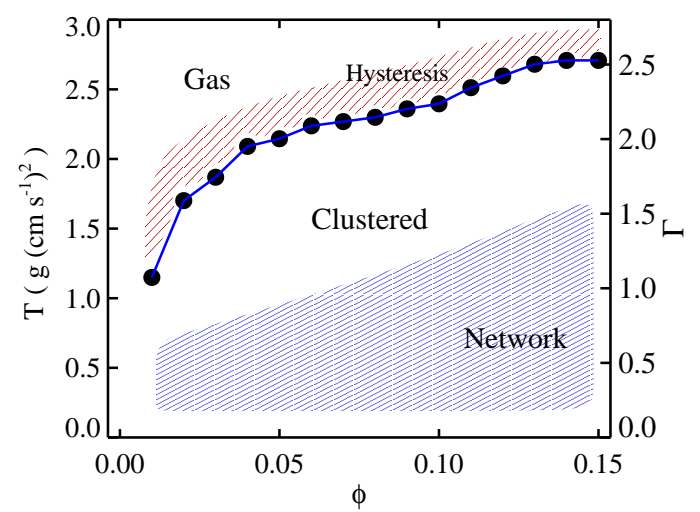

FIG. 2. (a) The phase diagram of temperature $T$ versus the covering fraction of the particles $\phi$. The plate acceleration $\Gamma$, scaled by gravity, is also shown for clarity. A gas phase consisting of single particles and short lived dimers and trimers are observed above a transition temperature $T_{s}$ that depends on $\phi$, shown by the solid points. To evaporate a cluster in the gas phase one must go past $T_{s}$ denoted by the hysteresis region. Below $T_{s}$, dimers and trimers act as seeds to the formation of compact clusters that coexist with single particles [Fig. If(d)]. If $T$ is rapidly quenched from the gas region to very low $T$ highly ramified networks of particles form [Fig. 1 $1(\mathrm{e})$ ].

above $T_{s}$, clusters show hysteretic behavior. This hysteresis depends on the ramping rate of $T$, and is not observed to disappear over laboratory time-scales. Therefore it appears that the observed phase transition is first order.

In the gas phase, we measure both the velocities of the non-magnetized glass particles and the magnetized steel particles and find that their distributions are indistinguishable. Figure 3 shows the distributions of velocities at $\phi=0.09$ for each particle species, viz. the gas of glass particles, the gas of magnetized steel particles (prior to and after nucleation of a cluster), and the particles within a cluster. The data shown corresponds to the system in Fig. I (d), although the results are the same for different values of $\phi$. When particles nucleate into clusters the fluctuations in the particle positions compared to the surrounding gas dramatically decreases, as shown by the narrow distribution in Fig. 3. However, the surrounding particles still have a temperature identical to that of the pure gas phase. The ratio of the system temperature to the cluster temperature $T_{c}$ is $T / T_{c} \approx 60$. This effect is inherent to the non-equilibrium nature of this system, where effects due to friction limit the thermal agitations expected in the constituent particles that comprise gels and polymers [17]. The existence of a finite measurable temperature in the clustered phase does imply however that particles are still mobilized, which can be seen by the substantial re-arrangements that occur during cluster growth.

By utilizing the Hoshen-Kopelmen 18 cluster identi- 


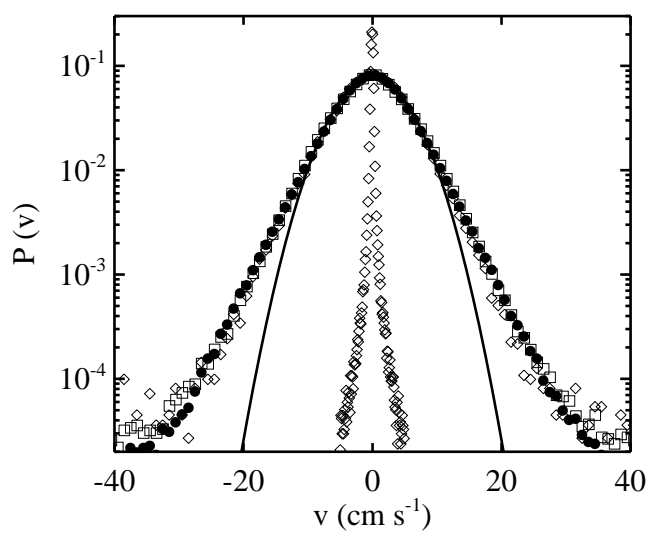

FIG. 3. The probability distribution functions $P(\mathbf{v})$ of the velocity components in the gas and clustered phases on a log linear scale. $(\diamond)$ The p.d.f.'s for the magnetic particles in both the gas and clustered phases. The narrow distribution is from particles within a cluster and the broad distribution (of the same symbol) is for the surrounding gas. ( $\square$ ) Magnetized, and $(\bullet)$ glass particles in the gas phase. The velocities are absolute velocities given in $\left(\mathrm{cm} \mathrm{s}^{-1}\right)$ not rescaled by the temperature. The solid line is a Gaussian fit to the magnetized gas distribution.

fication algorithm individual clusters that form at $T_{s}$ are identified. We are then able to track each clusters' size, center of mass, and radius of gyration. This method was also employed to measure the temperature of a single cluster and the surrounding gas. The average number of particles contained in a cluster $\left\langle n_{c}\right\rangle=n_{c} / n$, where $n$ is the total number of clusters formed, is plotted versus

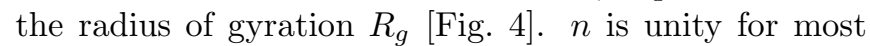
times, but may vary at very short times. This data was obtained for $1092 \mathrm{~s}$ immediately after $T$ was lowered to $T_{s}$. Using the scaling relation $n_{c} \sim R_{g}^{d}$ [19], we measure the compactness of the clusters. Figure 1 clearly shows a crossover in the dimensionality of the clusters. Clusters with $R_{g}<5.0 \sigma$ follow closely to the spatial dimension. As the number of particles per cluster increases with time the trend for the fractal dimension, $d$, at all $\phi$, is more consistent with $d=1.4 \pm 0.1$. This implies that clusters are initially more compact and become more extended as they grow. The change in dimensionality can be understood by observing the growth of a cluster. As particles join the cluster they do so by forming highly mobile chains that are tethered by one end to the surface of the cluster. If the free end of the chain is able to connect back onto the cluster an excluded area is established. During this process free particles may become trapped by the chains, which in time can produce rearrangements and re-opening of these regions. This can be seen by a close inspection of Fig. 1](d) and in Ref. [15].

Next we plot the cluster growth rates by measuring the number of free particles at $T_{s}$ for various $\phi$. We find that the average radius $r_{c}$ given by $n_{c}^{1 / d}(n=1$ for Fig. 国 over

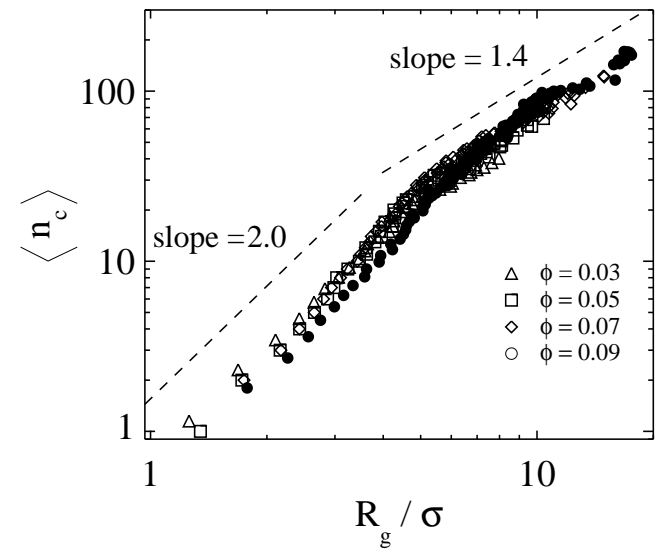

FIG. 4. Average cluster size $\left\langle n_{c}\right\rangle$ versus the radius of gyration $R_{g} / \sigma$. The scaling $\left\langle n_{c}\right\rangle=\alpha R_{g}^{d}$, where $\alpha=\pi^{2} / 2 \sqrt{3}$, shows a crossover at $R_{g} \sim 5 \sigma$ indicating that the clusters start more compact and become more extended.

all time) contained in a cluster grows linearly as the surrounding free particles are depleted to a saturation value determined by $\phi$. The initial linear growth of $r_{c}$ is consistent with the Wilson-Frenkel growth process of hard sphere colloidal crystals, where individual particles are absorbed onto the surface of a crystallite 20, although the mechanism is quite different. The change in the linear growth of $r_{c}$ to a more arrested rate is caused by two effects. As the clusters grow the number of free particles is reduced and therefore the growth rate must decrease as the system approaches steady state. Data sets shown in Fig. 5 (a) correspond to clusters that nucleate close to the center of the cell. Clusters are mobile and eventually drift to the edge of the cell [see Fig [1(d)]. Due to depletion forces 21], the cluster will never be able to return to the center of the cell once in contact with the sidewall. As a result, half of the surface is not absorbing free particles, therefore the growth rate is further decreased.

To characterize the temperature of each cluster and emphasize its difference from the system temperature, we measure the cluster temperature $T_{c}$ as a function of time at $\phi=0.09$ [Fig. 5(b)]. $T_{c}$ is not only observed to be much lower than the surrounding gas phase, but also observed to decrease with increasing cluster size.

Finally, we discuss the meta-stable network phase that is observed within the clustered phase, shown by the shaded region of the phase diagram [Fig 2]. This phase [see Figure [1(e)], directly demonstrates the inherent anisotropy of the potential described in Eq. 1. This phase is attained by quenching to a $T$ far below $T_{s}$ from the gas state. When the energy of the system is dramatically decreased in this manner, the particles quickly form chain configurations because locally the head-totail alignment is energetically most favorable. However, as discussed above, when the length of the chain is $\geq$ 4 particles, the energy of the chain can be further lowered by bending into a more compact structure. At low 


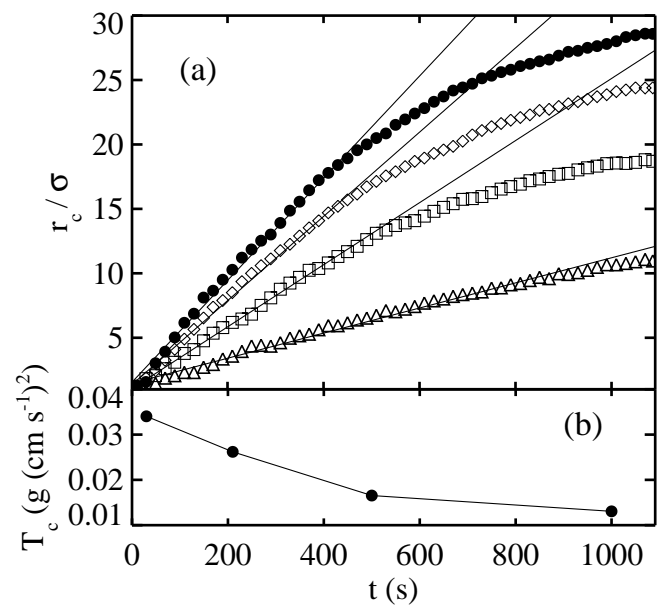

FIG. 5. (a) The radial growth rate $r_{c} / \sigma$ at $T=T_{s}$ for each $\phi$. The solid lines are fits to the data over a time when the cluster is not in contact with the wall. The apparent crossover in growth rate is due to a finite size effect. Linear growth is consistent with a Wilson-Frenkel growth process, where individual hard spheres are absorbed onto the cluster. Symbols for each $\phi=0.03(\triangle), 0.05(\square), 0.07(\diamond)$, and 0.09 $(\bullet)$. (b) The cluster temperature $T_{c}$ as a function of time for $\phi=0.09$. The temperature of the surrounding gas remains at a constant value of $T=T_{s}=2.2 \mathrm{~g}\left(\mathrm{~cm} \mathrm{~s}^{-1}\right)^{2}$

temperatures the probability of overcoming the potential barrier of bending is decreased, therefore we are able to observe the meta-stable state that is comprised mostly of string like objects for a longer period of time. It is selfconsistent that the deeper the quench, the longer the network phase survives, which is compounded even further by the dissipative interactions between particles. However, it is interesting that this phase shows similarities to recent Monte-Carlo simulations of quasi-2D dipolar hard spheres [22], where dissipation is not considered.

In summary, we visualize the nucleation of clusters in a non-equilibrium system by using magnetized granular materials. We have directly investigated the nature of the clusters formed by measuring their temperature, dimensionality and their growth rates. The temperature of the clusters is found to be considerably lower than the gas phase indicating a breakdown of equipartition. We also find that the phenomenology of the clusters is similar to that shown by recent work on dipolar hard sphere models, including a networked phase [10,22]. Although our system is weakly dissipative, it may provide still useful insights on the nature of phase-transitions in ideal dipolar system due to our ability to directly visualize and tune the particles in the experiments.

We thank C. Landee, H. Gould, G. Johnson, L. Colonna-Romano and T. Tlusty for fruitful discussions. This work was partially supported by National Science Foundation under Grant \# DMR-9983659 and Alfred P. Sloan Foundation.
[1] H. Jaeger, S. Nagel, and R. P. Behringer, Rev. Mod. Phys. 68, 1250 (1996).

[2] J. T. Jenkins and S. B. Savage, J. Fluid Mech. 130, 187 (1983); T. P. C. van Noije and M. H. Ernst, Granular Matter 1, 57 (1998).

[3] C. Bizon, et. al, Phys. Rev. E 60, 4340 (1999); E. L. Grossman, T. Zhou and E. Ben-Naim, ibid 55, 4200 (1997); A. Baldassarri, et. al, ibid 64, 011301 (2001).

[4] S. Warr, J. M. Huntley, and G. T. H. Jacques, Phys. Rev. E 52, 5583 (1995); W. Losert, D. Copper, J. Delour, A. Kudrolli, and J. P. Gollub, Chaos 9, 682 (1999); J. S. Olafsen and J. S. Urbach, Phys. Rev. E 60, R2468 (1999).

[5] A. Kudrolli and J. Henry, Phys. Rev. E 62, R1489 (2000); D. L. Blair and A. Kudrolli, Phys. Rev. E 64, 050301 (2001); K. Feitosa and N. Menon, Phys. Rev. Lett. 88, 198301 (2002).

[6] P. Tegzes, et al., Phys. Rev. E 60, 5823 (1999); A. Samadani and A. Kudrolli, Phys. Rev. E 64, 051301 (2001); I. S. Aranson, et. al, Phys. Rev. Lett. 84, 3306 (2000).

[7] M. E. van Leeuwen and B. Smit, Phys. Rev. Lett. 71, 3991 (1993).

[8] R.P. Sear, Phys. Rev. Lett. 76, 2310 (1996).

[9] P. J. Camp, J. C. Shelley, and G. N. Patey, Phys. Rev. Lett. 84, 115 (2000).

[10] T. Tlusty and S. A. Safran, Science 290, 1328 (2000).

[11] R. E. Rosensweig, Ferrohydrodynamics (Cambridge University Press, Cambridge, 1985).

[12] P. G. deGennes and P. A. Pincus, Phys. Kondens. Mater. 11, 189 (1970).

[13] H. Mamiya, I. Nakatani, and T. Furubayashi, Phys. Rev. Lett. 84, 6106 (2000).

[14] W. Wen, F. Kun, K. F. Pál, D. W. Zheng, and K. N. Tu, Phys. Rev. E 59, R4758 (1999).

[15] For a movie of this process please visit http://physics.clarku.edu/dipolar

[16] We have performed a calibration between the granular temperature $T$ and the acceleration amplitue $\Gamma$. We find a nearly linear correspondence that does not depend sensitively on the covering fraction $\phi$.

[17] A. G. Yodh et al, Phil. Trans. Roy. Soc. London A 359 921, (2001)

[18] J. Hoshen and R. Kopelman, Phys. Rev. B 14, 3438 (1976).

[19] $n_{c}=\alpha R_{g}^{d}$, where $\alpha$ is determined by the dimensionality and mass elements. We are 2D and use spheres (disks), therefore $\alpha=\pi^{2} / 2 \sqrt{3}$.

[20] B. J. Ackerson and K. Schätzel, Phys. Rev. E 52, 6448 (1995).

[21] P. D. Kaplan, J. L. Rouke, A. G. Yodh, and D. J. Pine, Phys. Rev. Lett. 72, 582 (1994).

[22] J. M. Tavares, J. J. Weis, and M. M. Telo da Gama, Phys. Rev. E 65, 061201 (2002). 